\title{
Efficient Routing Scheme Using Pivot Node in Wireless Sensor Networks
}

\author{
Jung-Seok Lee ${ }^{1}$, Jung-Pil Ryu ${ }^{2}$, and Ki-Jun Han ${ }^{1, *}$ \\ ${ }^{1}$ Department of Computer Engineering, Kyungpook National University, Daegu, Korea \\ leej.s@netopia.knu.ac.kr, kjhan@knu.ac.kr \\ ${ }^{2}$ Center for Technology fusion in Construct, Yonsei University, Seoul, Korea \\ goldmunt@yonsei.ac.kr
}

\begin{abstract}
We propose an efficient routing for wireless sensor networks, called pivot routing. Pivot routing establishes the data paths while query is propagated. Source node selects a data path and transmits data packets to the sink via pivot nodes. Because subsequent query and data packets are transmitted along pivot nodes, control message overhead decreases considerably. Network lifetime also increases since the energy consumption of communication is reduced at sensor node. Simulation results show good aspects of control overhead and network lifetime.
\end{abstract}

Keywords: Pivot routing, Pivot shifting, Energy efficient routing, Wireless sensor networks.

\section{Introduction}

Wireless sensor networks have been receiving a great deal of attention to be an infrastructure of ubiquitous computing environments. Many of routing protocols in wireless sensor networks have been introduced in the technical literature. In query based routing protocols, sink node floods query to networks and nodes search and construct routing path to delivery sensing data in response to the query of sink node. Finally, sink node sends query messages to source nodes after selecting path in the multiple paths and source nodes can start data delivery $[1,2,3]$.

We propose an efficient routing scheme which extends the network lifetime, called pivot routing. In pivot routing, since multiple data paths can be established when a query is flooded, control message overheads can be reduced. These benefits can prolong the network lifetime.

\section{Pivot Routing in Wireless Sensor Networks}

We propose a pivot routing in this section. In our scheme, since data paths are created between source nodes and sink while queries are propagated in the whole sensor networks, control overhead and total delay can be reduced as compared with the previous routing protocols.

\footnotetext{
* Correspondent author.
} 
We use the term pivot node to denote the sensors which construct the data paths. Once queries are propagated to sensor networks, selected pivot nodes construct the data paths toward the sink then sources just transmit the sensing information to a closest pivot node. We also have to consider the criteria to choose the pivot nodes. Several metrics can be candidates for selection, such as the hop counts from sink and the previous pivot node, the residual power, etc. In this paper, we only use the hop counts to select pivot nodes. We assume that the sensor nodes are not aware of their location, and already know the list of adjacent sensor nodes by HELLO exchange.

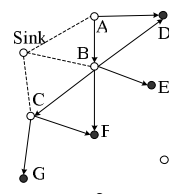

(a) Pivot selection

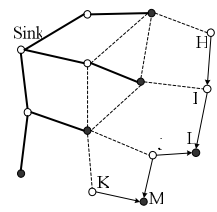

(b) Query complete

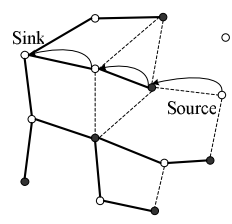

(c) Next forwarding/query drop

Fig. 1. The construction of data paths when pivot selection hop is 2

Initially, a sink sends queries for gathering the sensing information. A query message includes some mandatory information; \{query ID, pivot selection hop, prior pivot node ID, traverse node list $\}$. When a sensor node receives a query message, sensor nodes which being at distance of pivot selection hop are chosen as pivot nodes. Pivot selection hop $(>1)$ is assigned by a sink as a network parameter and included in query message. Whenever a query message is forwarded at a sensor, a sensor appends its own node id to the end of traverse node list in the query regardless of pivot selection. A pivot node maintains the node list table, named pivot table. Pivot table is composed of the traverse node list. Pivot nodes construct implicitly downstream path of the data to sink using primary traverse node list in the pivot table. A pivot node will forward the data to a previous pivot node with reference to pivot table. In order to transmit sensing data, previous pivot node id is stored at the all sensors which receive queries. Sensors between a sink and a pivot node would store the sink id.

In pivot routing, once a sensor is assigned as a pivot node, it always acts as a pivot node until it required that a pivot node is changed due to the specific condition, such as insufficient residual power or sensor failure. In order to prevent infinite flooding, each sensor node does not forward query which is already received.

Fig. 1 shows an example about the construction of data paths. Fig. 1(a) shows that a sink initially propagates queries to the sensor field. Even though B and C satisfy the pivot selection condition, these nodes can not be chosen as pivot node because these nodes are directly connected with sink node. The multiple paths to sink are very useful to overcome the transmission failure. When the query forwarding is completed, the data paths to the sink are accomplished, shown as fig. 1(b, c). In fig. 1(c), a source sends the sensing information immediately through pre-determined paths.

In pivot routing, the backup entries in the pivot table can be used to recover the link failure. Assume that link failure is occurred between node F and C, as shown fig. 2. F then looks up its own pivot table whether backup paths exist. $F$ has a new data path to sink via node B. Since a link failure is detected at a pivot node F, F finds 
easily the detour data path. It is different with link failure which is occurred at an intermediate node. In this instance, previous pivot id in the data is useful. An intermediate node sends link_failure message to the upstream pivot node. Upstream pivot node then selects an alternative path after looking up its pivot table. If no alternative path is found, a link_failure message is sent again to the upstream pivot node. Because pivot routing can maintain the multiple paths, it has little possibility that the pivot node closest to the source receives link_failure. In worst case, source node sends data to sink again as fig. 2(c), but such case is not likely to occur, because sensor nodes are distributed with high density.

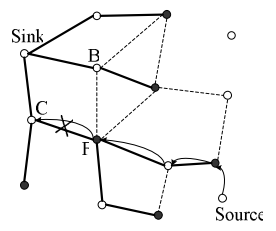

(a) Transmission failure

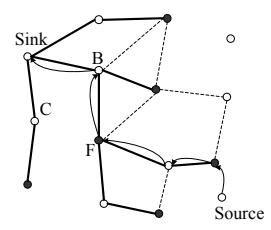

(b) Local repair

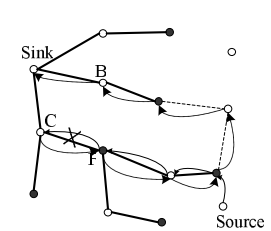

(c) Worst case of data delivery

Fig. 2. Recovery from transmission failure

\section{Performance Evaluation}

We evaluate pivot routing scheme through simulation. Simulation results are compared with TTDD in stationary sink situation. The size of sensor networks is $2000 \times 2000\left(\mathrm{~m}^{2}\right)$. The number of sensor nodes is increased from 100 to 500 nodes random distribution manner. Source nodes are randomly chosen, and the radius of radio transmission range is given $300(\mathrm{~m})$. A source generates a data packet per second. Each simulation runs for 200 seconds, each result is averaged over several repetitions. Sink broadcasts a query only once after the sensor network initialized. In our simulations, we assume that there is no unidirectional links and each nodes know its own neighbors using HELLO exchange.

We use two performance metrics to validate a proposed pivot routing. The normalized packet overhead is the ratio of the number of control packet to the total number of packets in whole sensor networks. This metric is directly concerned with overall network lifetime. The network lifetime means that how many nodes are alive.

Fig. 3 shows the simulation results. Normalized packet overhead indicates how many packets are traveling in sensor networks except data packets.

In pivot routing, a sink node forwards only one query packet. Although the number of source node increases, control message overhead decreases more and more, because the number of query message does not increases. Because the number of DAM message increases in proportion to the increment of the source nodes in TTDD, control message overhead rapidly increases in the whole sensing networks.

Fig. 3(b) shows that difference of control packets influence on the overall network lifetime because of the energy consumption for exchanging the control message with each other sensor nodes. 


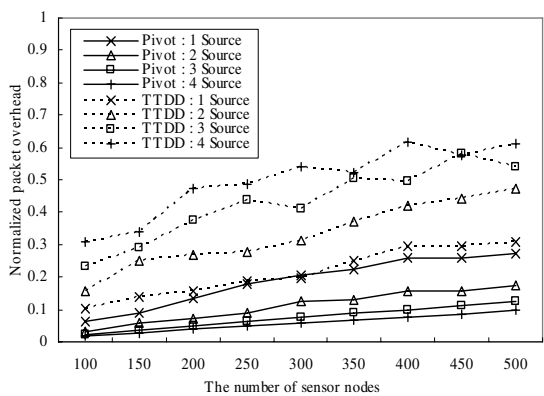

(a) Normalized packet overhead

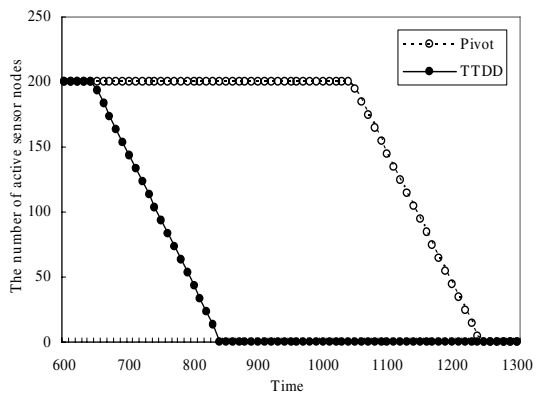

(b) Network lifetime

Fig. 3. Simulation results

Because the number of query message from sinks is same even though different pivot selection hop, the control message overhead keeps same pattern. Data packets almost traverse the same path, nothing but the hop distance among pivot nodes is just longer. Although pivot selection hop is varied, it does not affect on the number of control message as well as network lifetime.

\section{Conclusions and Future Works}

In this paper, we propose pivot routing algorithm to reduce the control message overhead and prolong the network lifetime. In our scheme, pivot nodes are determined when queries go through the whole networks just one time. Pivot nodes play the role of designated router and multiple paths are created between pivot nodes. Simulation results show that pivot routing provides lower control overhead and prolongs the network lifetime than TTDD.

We are going to consider the sink mobility and use other parameters to determine the pivot nodes (residual power, neighbor density and so on) in the future.

\section{Reference}

1. J. N. Al-Karaki and A. E. Kamal, "Routing techniques in wireless sensor networks: a survey," IEEE Wireless Communications, vol. 11, pp. 6-28, 2004.

2. K. Akkaya and M. Younis, "A survey on routing protocols for wireless sensor networks," Ad Hoc Networks, Vol.3, Issue 3, pp. 325-349, May 2005.

3. C. Intanagonwiwat, R. Govindan, D. Estrin, J. Heidemann and F. Silva, "Directed diffusion for wireless sensor networking," IEEE/ACM Transactions on Networking, Vol.11, Issue 1, pp.2-16, Feb.2003.

4. H. Luo, F. Ye, J. Cheng, S. Lu and Lixia Zhang, "TTDD: Two-Tier Data Dissemination in Large-Scale Wireless Sensor Networks,” Wireless Networks, Vol.11, pp.161-175, 2005. 